Received: 2019.03.30 Accepted: 2019.05.14 Published: 2019.09 .07
Authors' Contribution: Study Design A Data Collection B Statistical Analysis C Data Interpretation D Manuscript Preparation E Literature Search F Funds Collection G

\section{Anticancer Action of Psilostachyin-A in 5-Fluorouracil-Resistant Human Liver Carcinoma are Mediated Through Autophagy Induction, G2/M Phase Cell Cycle Arrest and Inhibiting Extracellular-Signal-Regulated Kinase/Mitogen Activated Protein Kinase (ERK/MAPK) Signaling Pathway}

\author{
CDE Jun Liu \\ $A B C$ Yan Liu \\ BCD Yan Liu \\ EF Lei Huang \\ BCD Guoliang Wang \\ DEF Jun Wang \\ DEF Xiangang Xu \\ CEF Chengxian Shi \\ ACDG Jianzhao Huang
}

Department of Hepatobiliary Surgery, Guizhou Provincial People's Hospital, Guiyang, Guizhou, P.R. China
Corresponding Author: Source of support:

Background: Liver cancer is one of the most common malignancies around the world and one of the major causes of cancer related mortality. The objective of this study was to evaluate the anticancer effect of the natural compound psilostachyin-A on 5-fluorouracil-resistant human liver carcinoma cells and its effects on autophagy, cell cycle, caspase activation, and the ERK/MAPK signaling pathway.

Material/Methods: Cell Counting Kit 8 (CCK-8) assay was used to evaluate the effects on HepG2 cell viability at different doses of psilostachyin-A. Cell cycle analysis was performed using flow cytometry, and Transwell assay was used to check effects on cell invasion. Transmission electron microscopic studies were done to evaluate autophagy induced by psilostachyin-A, and the western blot method was carried out to evaluate the effects on autophagy and the ERK/MAPK signaling pathway.

Results: CCK-8 assay revealed that the psilostachyin-A reduced the cell viability of HepG2 cancer cells in a dose dependent manner. Psilostachyin-A also reduced the colony forming potential of HepG2 cells, concentration dependently. The IC50 of psilostachyin was found to be $25 \mu \mathrm{M}$. The anticancer effects of psilostachyin-A were due to the induction of autophagy which was accompanied by enhancement of LC3B II expression. Psilostachyin also caused cell cycle arrest by enhancing the accumulation of HepG2 cells in the G2/M phase. Transwell assay showed that psilostachyin-A suppressed the invasion of HepG2 cells. The results also showed that psilostachyin-A could block the ERK/MAPK pathway, indicative of the cytotoxic effects of psilostachyin-A on liver cancer.

Conclusions: These preliminary observations suggested that psilostachyin-A might prove beneficial in the treatment of liver cancer.

MeSH Keywords:

Autophagy • Carcinoma, Hepatocellular • Caspase 1 • Flow Cytometry

Full-text PDF: https://www.medscimonit.com/abstract/index/idArt/916635

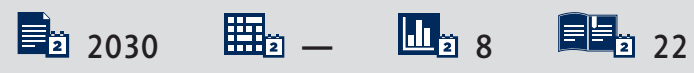




\section{Background}

The use of plant extracts or plant derived products dates to ancient times. People have used plants for their primary health care needs since times immemorial [1]. Subsequently, phytochemicals have attained the attention of researchers around the world for their health promoting benefits. Different traditional systems of medicine utilize plants for the treatment of deadly diseases [2]. Plants have the capability to synthesize diverse chemical scaffolds for their own defense. These metabolites are known as secondary metabolites [3]. Sesquiterpene psilostachyin-A is a sesquiterpene lactone and is believed to exhibit tremendous pharmacological potential [4]. Sesquiterpene lactones are considered potent anticancer agents and many sesquiterpene lactones have been studied in clinical trials [5]. Nonetheless, there is not a single report on the anticancer activity of the sesquiterpene lactone psilostachyin-A against the liver cancer cells. This study was therefore undertaken to investigate the anticancer effects of psilostachyin-A against 5-fluorouracil-resistant human liver carcinoma cells and the underlying mechanisms for these anticancer effects of liver carcinoma cells. Liver cancer is a fatal malignancy and believed to be the second prevalent cause of cancer-related mortality worldwide [6]. The liver cancer incidence has remarkably increased over the last few decades, and the identification of novel chemotherapeutic agents are urgently needed [7]. Herein, we, for the first-time, report that psilostachyin-A selectively targets cancer cells via induction of autophagy and G2/M cell cycle arrest. One of the important features of psilostachyin-A is that it can inhibit the invasion of the HepG2 cells. Although a lot of progress has been made with regard to development of anticancer drugs, these drugs have limited curative tendencies, which poses a clinical obstacle in treatment. This limited curative tendency is mainly due to the development of multidrug resistance induced by conventional anticancer drugs. It has been reported that multidrug resistance is mainly due to overexpression of certain efflux pumps, DNA damage repair, autophagy induction, etc. [8]. The main objective of this study was to investigate the anticancer potential of psilostachyin-A along with evaluating its effects on autophagy, cell cycle arrest, and the ERK/MAPK signaling pathway. In this study, psilostachyin-A was found to block the ERK/MAPK pathway in a concentration dependent manner. Hence, psilostachyin-A was found to be an important sesquiterpene lactone and exhibited the potential to inhibit the growth of liver cells. We strongly believe that psilostachyin-A might act as an important lead molecule in liver cancer drug discovery paradigms and warrants further studies.

\section{Material and Methods}

\section{Cell viability assay}

The HepG2 cell line and AML12 cell line were procured from Cell Bank of Chinese Academy of Science (Shanghai, China). The cells were kept in Dulbecco's modified Eagle's medium (DMEM). The HepG2 cells were subjected to treatment with different concentrations $(0,2.5,5,10,20,40,80,160$, and $320 \mu \mathrm{M})$ of psilostachyin-A and cultured in 96-well plates for 24 hours. Afterwards, $10 \mu \mathrm{L}$ of Cell Counting Kit 8 (CCK-8, Dojindo Laboratories, Kumamoto, Japan) was added to the culture plates, and the plates were incubated for 2 hours at $37^{\circ} \mathrm{C}$ in humidified $95 \%$ air, and $5 \% \mathrm{CO}_{2}$. The absorbance was measured at $450 \mathrm{~nm}$ using a microplate Reader (Bio-Rad, Hercules, CA, USA).

\section{Colony formation assay}

The method of Gupta et al. [9] was used to assess the effects of psilostachyin-A on the colony forming potential of the HepG2 cells. Briefly, the HepG2 cells were subjected to treatment with psilostachyin-A $(0,12.5,25$, and $50 \mu \mathrm{M})$ for 24 hours. Following this, the HepG 2 cells were then seeded in the 6 -well plates and incubated at $37^{\circ} \mathrm{C}$ for 1 week. The colonies so developed were stained with crystal violet $(0.1 \%)$ and counted under an inverted microscope (Olympus Corporation, Japan).

\section{Cell cycle analysis}

The HepG 2 cells treated with $0.25 \%$ trypsin were centrifuged at $1000 \mathrm{rpm}$ for 5 minutes to remove the DMEM medium. The cells were washed with phosphate-buffered saline (PBS), then the supernatant was discarded, and the cells were collected simultaneously. They were washed twice with ice cold PBS and fixed in $80 \%$ ethanol at $4^{\circ} \mathrm{C}$ overnight. After washing 3 times with PBS, the cells were suspended in $0.1 \mathrm{mg} / \mathrm{mL}$ propidium iodide $(\mathrm{PI})$ at $37^{\circ} \mathrm{C}$ for half an hour without light. Cell cycle was detected by FACS Calibur flow cytometer (BD Biosciences, USA) and expressed as the percentage of cells in each phase of the cell cycle.

\section{Transmission electron microscopy}

The HepG 2 cells were treated with psilostachyin-A at the concentrations of $0,12.5,25$, and $50 \mu \mathrm{M}$ concentrations of psilostachyin-A. A solution of $4 \%$ glutaraldehyde and $0.05 \mathrm{M}$ sodium cacodylate was then used to fix the cells. This was followed by post-fixation in $0 \mathrm{SO} 4$ (1.5\%), and then dehydration in alcohol. Afterwards, the cells flat embedded in Epon 812 and finally observed under JEM 1010 transmission electron microscope (JEOL, Peabody, MA, USA). 
A

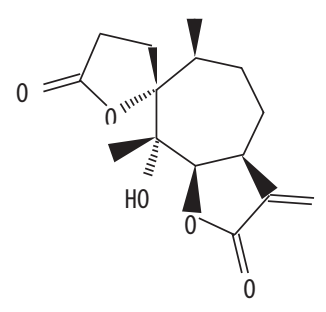

B

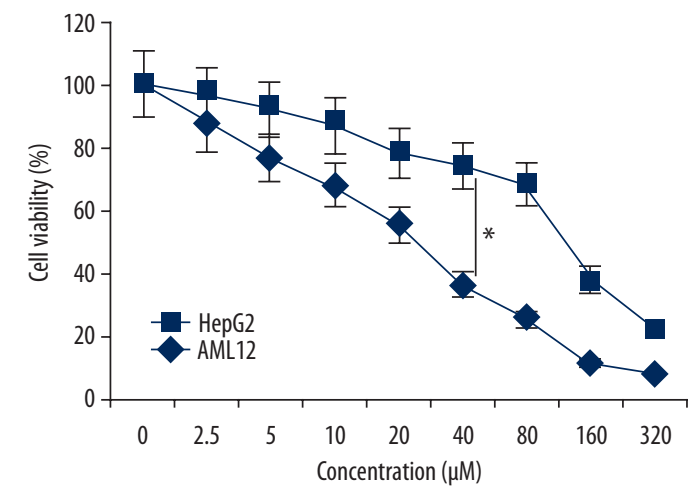

Figure 1. (A) Structure of psilostachyin-A. (B) Effect of psilostachyin-A on the viability of HepG2 and AML12 cells as determined by Cell Counting Kit 8 assay. Psilostachyin-A showed more cytotoxicity towards cancer cells rather than normal AML12 cells. The values are mean of 3 replicates \pm standard deviation $\left({ }^{*} P<0.05\right)$.

\section{Transwell assay}

The effects of psilostachyin-A on the invasion ability of HepG2 cells was determined by Transwell chambers ( $8 \mathrm{~mm}$ pore size, Corning, NY, USA) with Matrigel (Millipore, Billerica, USA). Around $200 \mathrm{~mL} \mathrm{HepG} 2$ cell cultures were placed onto the upper chambers and only medium was placed in the bottom wells. After 24 hours of incubation, the cells were removed from the upper chamber and the cells that invaded via the chambers were subjected to fixation with methyl alcohol and subsequently stained with crystal violet. Inverted microscope was used to count the number of invaded cells at $200 x$ magnification.

\section{Western blot analysis}

The HepG2 cells were then lysed in lysis buffer containing the protease inhibitor. Around $45 \mu \mathrm{g}$ of proteins from each sample were subjected to separation $10 \%$ and followed by transferring it to polyvinylidene difluoride (PVDF) membrane. Next, fat-free milk was used to block the membrane at room temperature for 1 hour. Thereafter, the membranes were treated with primary antibodies at $4^{\circ} \mathrm{C}$ for overnight. Subsequently, the membranes subjected to incubation with secondary antibodies. Finally, the signal was detected by Odyssey Infrared Imaging System. Actin was used as control for normalization.

\section{Statistics}

The results are presented as mean \pm standard deviation values from 3 independent experiments. Differences between the groups were examined by Student's $t$-test using SPSS 17.0 software (SPSS, Inc., Chicago, IL, USA). $P<0.05$ was considered to indicate a statistically significant difference.

\section{Results}

\section{Psilostachyin-A suppressed the proliferation of liver cancer} cells

To find out if psilostachyin-A suppressed the proliferation of the HepG2 cells, the CCK-8 cell viability assay was performed. It was revealed that upon treatment with psilostachyin-A (Figure $1 \mathrm{~A}$ ) the proliferation rate of 5 -fluorouracil-resistant HepG2 cells was remarkably decreased. An IC50 of $25 \mu \mathrm{M}$ was observed for psilostachyin-A against the HepG2 cells (Figure 1B). However, the effects of psilostachyin-A were comparatively lower against the normal AML12 hepatocytes which showed a higher IC50 value.

\section{Psilostachyin-A suppressed the colony formation of liver cancer cells}

The colony formation assay was performed to assess the effects of psilostachyin-A HepG 2 colony forming potential. The HepG2 cells were treated with $0,12.5,25$, and $50 \mu \mathrm{M}$ and incubated at $37^{\circ} \mathrm{C}$ for 1 week. It was found that the psilostachyin-A suppressed the colony development potential of the HepG2 cells in a concentration dependent manner (Figure 2). As the concentration of psilostachyin-A was increased, the potential of the HepG2 cells to form colonies decreased.

\section{Psilostachyin-A induced autophagy in the HepG2 cells}

Electron microscopic analysis of the psilostachyin-A treated HepG 2 cells showed that psilostachyin-A caused the development of the autophagosomes in the HepG2 cells (Figure 3). The frequency of autophagosomes in HepG2 cells was increased with increase in the concentration of the psilostachyin-A. Moreover, psilostachyin-A caused increase in the expression 

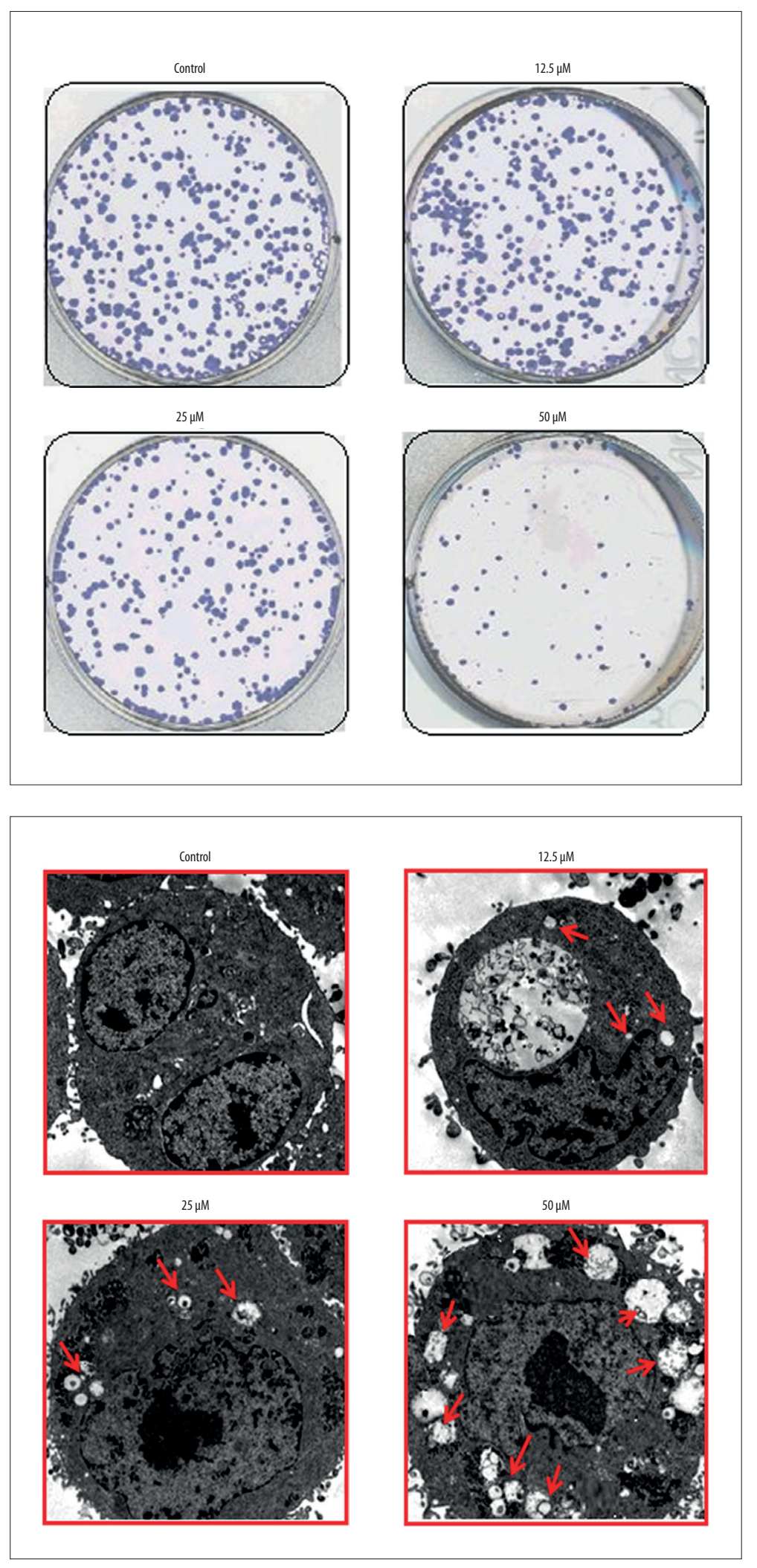

Figure 2. Effect of on the colony formation of the psilostachyin-A treated HepG2 cells as determined by microscopy. The experiments were performed in triplicate. Psilostachyin-A decreased cancer colony formation dose-dependently.

Figure 3. Psilostachyin-A induces autophagy in the HepG2 cells as indicated by electron microscopy. The experiments were performed in triplicate. Psilostachyin-A led to the formation of autophagosomes in a dose-dependent manner. 


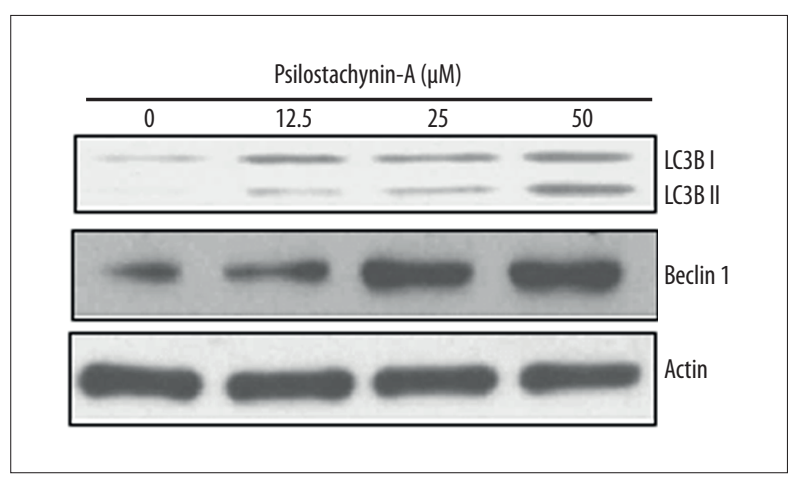

Figure 4. Effect of psilostachyin-A on the expression of LC3 and Beclin-1 in HepG 2 cells as determined by western blot analysis. The experiments were performed in triplicate. psilostachyin-A caused increase in the expression of LC3B-II as well as the Beclin-1 expression. of LC3B-II as well as the Beclin-1 expression. However no significant changes were observed in the expression of LC3B-I in the HepG2 cells (Figure 4).

\section{Psilostachyin-A induced $G_{2} / M$ arrest of the HepG2 cells}

The distribution of the psilostachyin-A treated HepG2 cells was determined by flow cytometry. The results showed that psilostachyin-A caused remarkable increase in the percentage of the HepG 2 cells. The percentage of the HepG 2 cells was found to be $12.5 \%, 41.3 \%, 67.1 \%$, and $72.5 \%$ at $0,12.5,25$, and $50 \mu \mathrm{M}$ concentrations respectively of psilostachyin-A, which was indicative of G2/M arrest of the HepG2 cells (Figure 5). Western blot analysis revealed that psilostachyin-A caused a decline of cyclin B1 and CDK1 expression in a concentration dependent manner (Figure 6).

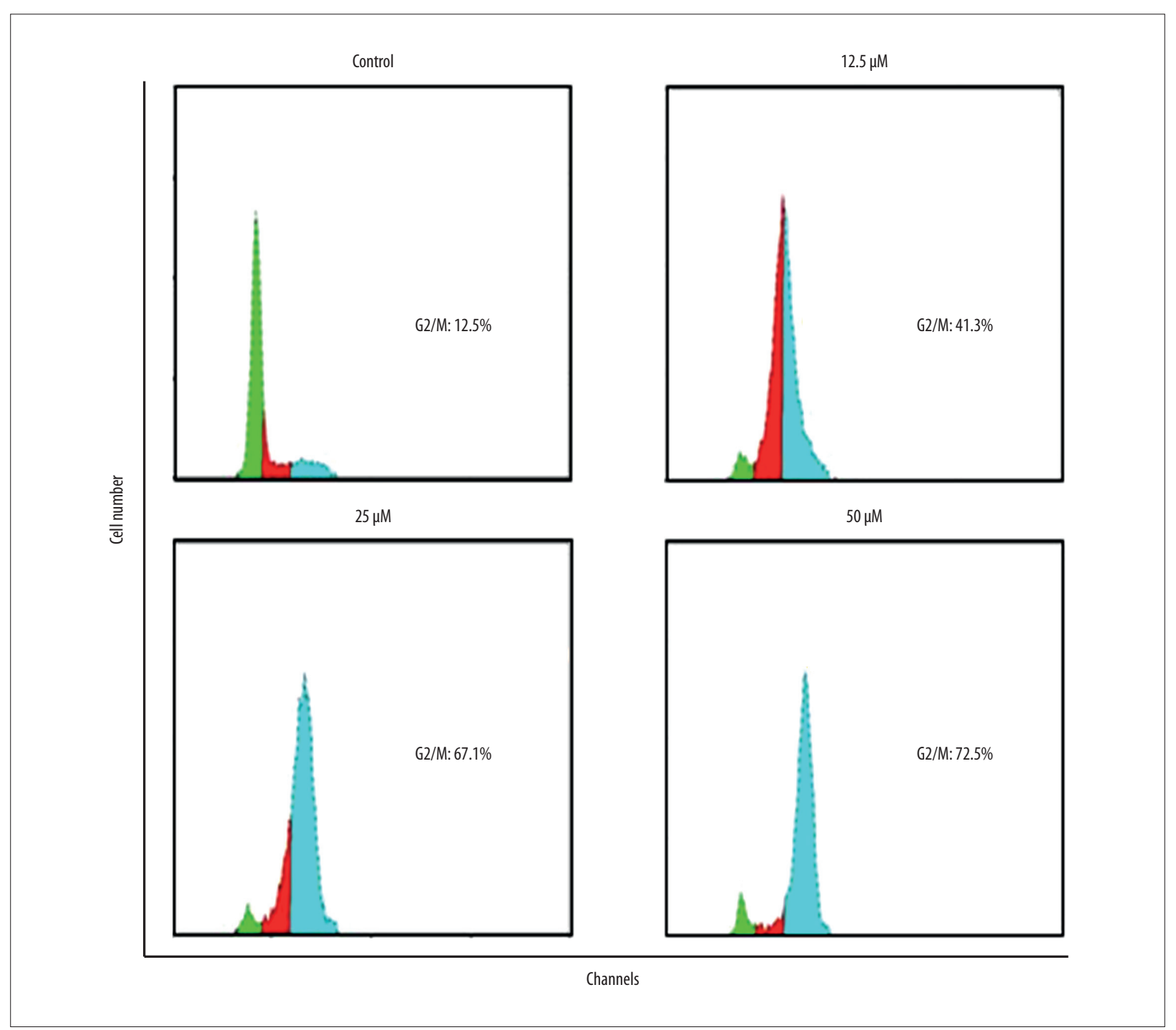

Figure 5. Effect of psilostachyin-A on the distribution of the HepG2 cells in different cell cycle phases at indicated concentrations. The experiments were performed in triplicate. psilostachyin-A caused remarkable increase in the percentage of the HepG2 cells.

\section{5}

Indexed in: [Current Contents/Clinical Medicine] [SCI Expanded] [ISI Alerting System] [ISI Journals Master List] [Index Medicus/MEDLINE] [EMBASE/Excerpta Medica] [Chemical Abstracts/CAS] 


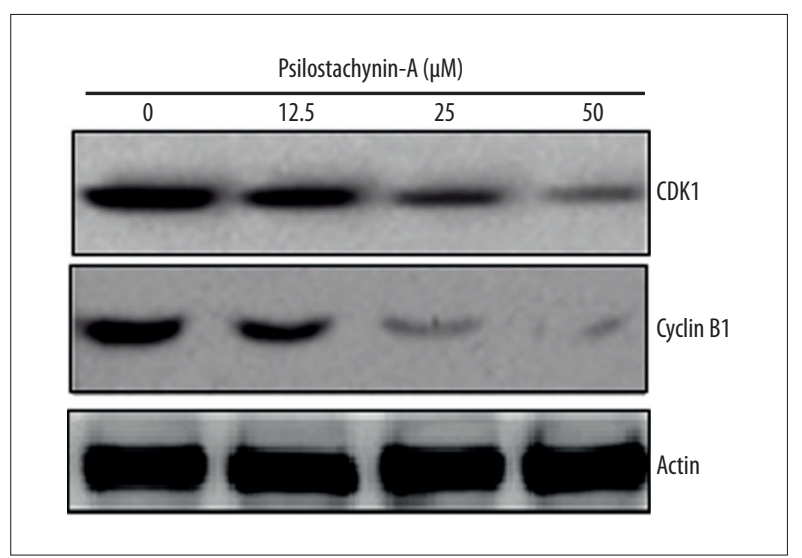

Figure 6. Effect of psilostachyin-A on the expression of CDK1 and cyclin B1 in HepG2 cells at indicated concentrations as depicted by western blot analysis. The experiments were performed in triplicate. Psilostachyin-A caused decline of cyclin B1 and CDK1 expression in a concentration dependent manner.

\section{Psilostachyin-A inhibited invasion of the HepG2 cells}

The effects of psilostachyin-A at the concentrations of $0,12.5$, 25 , and $50 \mu \mathrm{M}$ on the invasion of the HepG2 cells were observed by performing the Transwell assay. The results showed that psilostachyin-A suppressed the invasion of the HepG2 cells in a dose dependent manner (Figure 7).

\section{Psilostachyin-A inhibited the MAPK/ERK signaling pathway in HepG2 cells}

The effects of psilostachyin-A were also investigated on the MAPK/ERK signaling pathway and the results of the western blot analysis showed that psilostachyin-A inhibited the phosphorylation of p38 and ERK in a dose dependent manner (Figure 8). Nonetheless, no effects were observed on the total MAPK and ERK pathway.

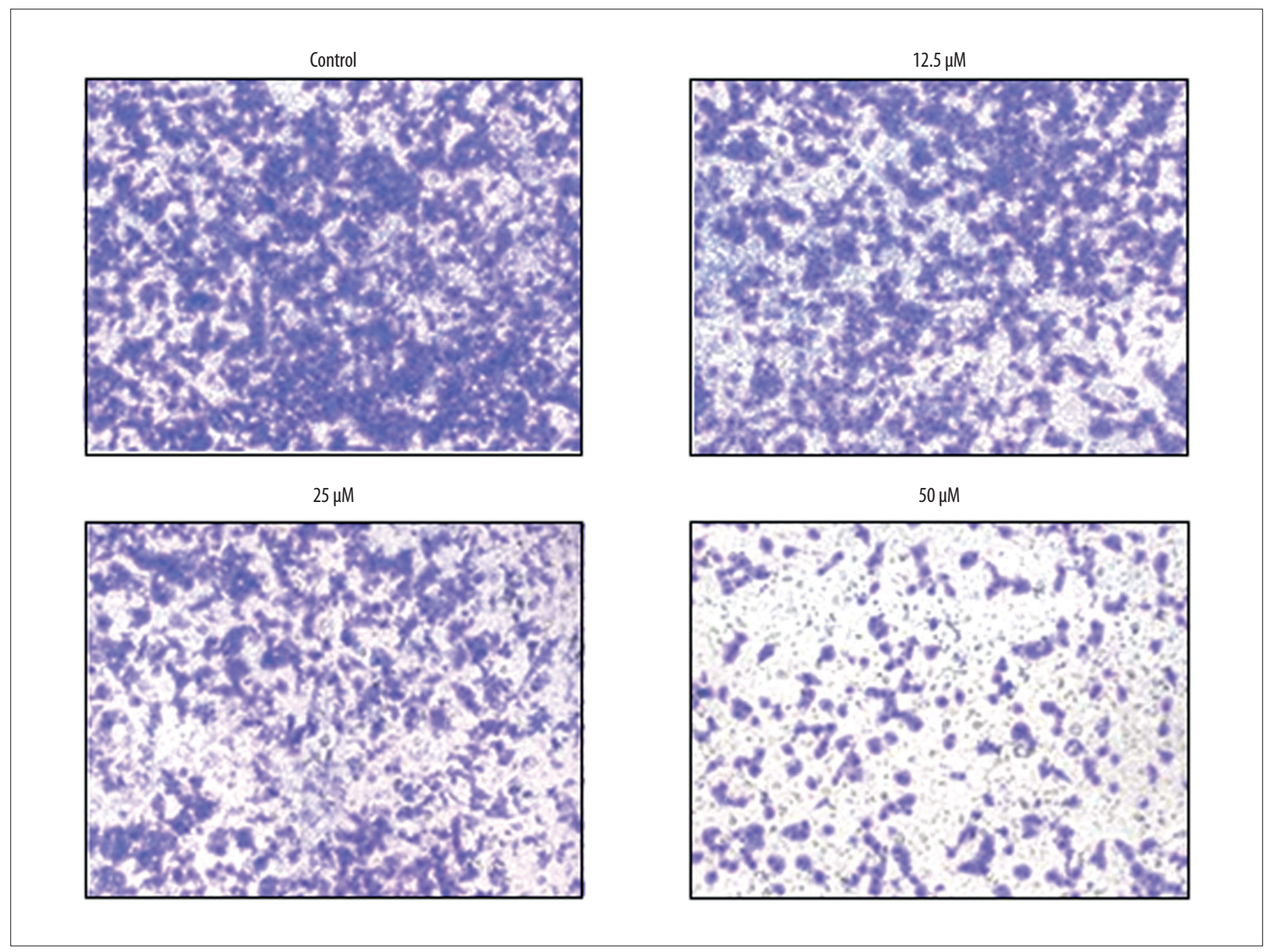

Figure 7. Effect of psilostachyin-A on the invasion of the HepG2 cells at indicated concentrations as determined by Transwell assay. The experiments were performed in triplicate. It caused a significant decrease in cancer cell invasion. 


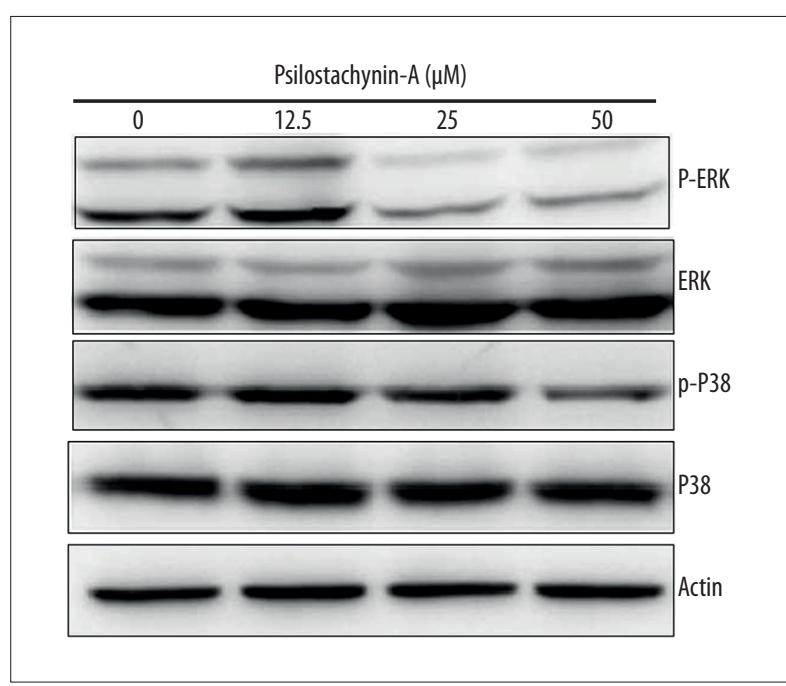

Figure 8. Inhibition of the phosphorylation of p38 and ERK by psilostachyin-A at indicated concentrations as depicted by western blot analysis. The experiments were performed in triplicate.

\section{Discussion}

Liver cancer is one of the fatal malignancies responsible for significant number of human deaths [10]. The prevalence of liver cancer is increasing constantly and is expected to increase enormously in the years to come [11]. Compounds of plant origin have amazing potential to curb the growth and development of cancers [12]. Plant derived molecules inhibit the proliferation of cancer cells via different mechanisms such as apoptosis, autophagy and arrest of the cancer cells at different cell cycle phases [13]. Some anti-cancerous molecules block the signaling cascade that is generally aberrantly hyperactivated in cancer cells, and others activate the signalling pathways that are generally deactivated in cancer cells [14]. Additionally, molecules of plant origin are considered comparatively safer for human consumption. So, it is imperative to mention that plant derived anticancer drugs might exhibit negligible adverse effects on the overall health of patients [15]. This study was undertaken to examine the anticancer effects of psilostachyin-A against the human HepG2 cells as well as normal human liver cells. It was found that psilostachyin-A suppressed the proliferation rate of the 5 -fluorouracil-resistant human liver cancer cells. However, the anticancer effects of psilostachyin-A were found to be specific to HepG2 cells as shown by very high IC50 observed against normal hepatocytes. Moreover, psilostachyin-A also decreased the colony forming potential of HepG2 cells. These observations confirm previous studies wherein many sesquiterpene lactones have been shown to cause a decrease in the proliferation of the cancer cells, for example, sesquiterpene lactone parthenolide has been reported to suppress the growth of renal carcinoma cells [16]. To find out the underlying mechanism for the anticancer activity of psilostachyin-A, an electron microscopic analysis of psilostachyin-A treated HepG2 cells was carried out and we found that psilostachyin-A caused the development of autophagosomes in HepG2 cells, indicative of autophagy. Furthermore, psilostachyin-A also caused enhancement in the expression of LC3B-II and Beclin-1, which was concentration dependent. Previous studies have also shown that sesquiterpene lactones, such as isodeoxyelephantopin, induce autophagy in lung cancer cells [17]. Sesquiterpene lactones have also been shown to cause cell cycle arrest of cancer cells [18]. Herein, we found that psilostachyin-A caused the arrest of HepG2 cancer cells via induction of autophagy. Psilostachyin-A also caused the inhibition in the invasion of the HepG2 cells indicative of the anti-metastatic potential of psilostachyin-A. MAPK/ERK cascade has been shown to be activated in cancer cells and might have therapeutic implications [19]. Herein, we found that psilostachyin-A could, concentration dependently, block this pathway.

Even though a lot of improvements have been made regarding development of anticancer drugs, these drugs have limited curative tendency which poses a clinical obstacle in treatment. This limited curative tendency is mainly due to development of multidrug resistance induced by conventional anticancer drugs. It has been reported that multidrug resistance is mainly due to overexpression of certain efflux pumps, DNA damage repair, autophagy induction, etc. Hence, focus should be reoriented more on exploring the molecular mechanisms behind chemoresistance with an aim to develop novel therapeutic targets and diagnostic biomarkers [20-22].

\section{Conclusions}

The outcomes of the present study indicated that psilostachyin-A exerts significant anticancer effects on the human liver cancer cells. The anticancer effects of psilostachyin-A are mainly because of autophagy induction and cell cycle arrest. Taken together, psilostachyin-A might be utilized in the development of chemotherapy for liver cancer and deserves further studies.

\section{Conflict of interest}

None. 


\section{References:}

1. Cragg GM, Newman DJ: Plants as a source of anti-cancer agents. J Ethnopharmacol, 2005; 100(1-2): 72-79

2. Gordaliza M: Natural products as leads to anticancer drugs. Clin Transl Oncol, 2007; 9(12): 767-76

3. Cragg GM, Newman DJ, Snader KM: Natural products in drug discovery and development. J Nat Prod, 1997; 60(1): 52-60

4. Mabry TJ, Miller HE, Kagan HB, Renold W: The structure of psilostachyin, a new sesquiterpene dilactone from ambroisia psilostachya. Tetrahedron, 1966; 22(3): 1139-46

5. Ghantous A, Gali-Muhtasib $\mathrm{H}$, Vuorela $\mathrm{H}$ et al: What made sesquiterpene lactones reach cancer clinical trials? Drug Discov Today, 2010; 15(15-16): 668-78

6. El-Serag HB, Rudolph KL: Hepatocellular carcinoma: Epidemiology and molecular carcinogenesis. Gastroenterology, 2007; 132(7): 2557-76

7. Gomaa Al, Khan SA, Toledano MB et al: Hepatocellular carcinoma: Epidemiology, risk factors and pathogenesis. World J Gastroenterol, 2008; 14(27): 4300

8. Wen L, Liang C, Chen E et al: Regulation of multi-drug resistance in hepatocellular carcinoma cells is TRPC6/calcium dependent. Sci Rep, 2016; 6 : 23269

9. Gupta D, Crosby ME, Almasan A, Macklis RM: Regulation of CD20 expression by radiation-induced changes in intracellular redox status. Free Radic Biol Med, 2008; 4: 614-23

10. Bruix J, Sherman M: Management of hepatocellular carcinoma: An update. Hepatology, 2011; 53(3): 1020-22

11. El-Serag HB, Marrero JA, Rudolph L, Reddy KR: Diagnosis and treatment of hepatocellular carcinoma. Gastroenterology, 2008; 134(6): 1752-63

12. Reddy LA, Odhav B, Bhoola KD: Natural products for cancer prevention: a global perspective. Pharmacol Ther, 2003; 99(1): 1-3
13. Prietsch RF, Monte LD, Da Silva FA et al: Genistein induces apoptosis and autophagy in human breast MCF-7 cells by modulating the expression of proapoptotic factors and oxidative stress enzymes. Mol Cell Biochem, 2014; 390(1-2): 235-42

14. Liu Y, Jiang YG: Podocalyxin promotes glioblastoma multiforme cell invasion and proliferation via $\beta$-catenin signaling. PLoS One, 2014; 9(10): e111343

15. Leung HW, Lin CJ, Hour MJ et al: Kaempferol induces apoptosis in human lung non-small carcinoma cells accompanied by an induction of antioxidant enzymes. Food Chem Toxicol, 2007; 45(10): 2005-13

16. Oka D, Nishimura K, Shiba M et al: Sesquiterpene lactone parthenolide suppresses tumor growth in a xenograft model of renal cell carcinoma by inhibiting the activation of NF-KB. Int J Cancer, 2007; 120(12): 2576-81

17. Wang $\mathrm{Y}$, Zhang J, Huang $\mathrm{ZH}$ et al: Isodeoxyelephantopin induces protective autophagy in lung cancer cells via Nrf2-p62-keap1 feedback loop. Cell Death Dis, 2017; 8(6): e2876

18. Rozenblat $S$, Grossman $S$, Bergman $M$ et al: Induction of $G 2 / M$ arrest and apoptosis by sesquiterpene lactones in human melanoma cell lines. Biochem Pharmacol, 2008; 75(2): 369-82

19. Mirzoeva OK, Das D, Heiser LM et al: Basal subtype and MAPK/ERK kinase (MEK)-phosphoinositide 3-kinase feedback signaling determine susceptibility of breast cancer cells to MEK inhibition. Cancer Res, 2009; 69(2): 565-72

20. Lohitesh K, Chowdhury R, Mukherjee S: Resistance a major hindrance to chemotherapy in hepatocellular carcinoma: An insight. Cancer Cell Int, 2018; 18: 44

21. Sukowati Ch, Rosso N, Crocè LS, Tiribelli C: Hepatic cancer stem cells and drug resistance: Relevance in targeted therapies for hepatocellular carcinoma. World J Hepatol, 2010; 2(3): 114-26

22. Li H, Zhang X, Wang W: Anticancer activity of 5, 7-dimethoxyflavone against liver cancer cell line HepG2 involves apoptosis, ROS generation and cell cycle arrest. Afr J Tradit Complement Altern Med, 2017; 14(4): 213-20 